\title{
Biomarkers of diagnosis and resolution of food allergy
}

\author{
$\mathrm{Ru}_{-\mathrm{Xin}}$ Foong $^{1}$ and Alexandra Santos ${ }^{1}$ \\ ${ }^{1}$ King's College London
}

June 15, 2020

\begin{abstract}
Food allergy is increasing in prevalence, affecting up to $10 \%$ of children in developed countries. Food allergy can significantly affect the quality of life and well-being of patients and their families; therefore, an accurate diagnosis is of extreme importance. Some food allergies can spontaneously resolve in 50-60\% of cow's milk and egg allergic, $20 \%$ of peanut allergic and $9 \%$ of tree nut allergic children by school age. For that reason, food allergic status should be monitored over time to determine when to reintroduce the food back into the child's diet. The gold-standard to confirm the diagnosis and the resolution of food allergy is an oral food challenge; however, this involves a risk of causing an acute allergic reaction and requires clinical experience and resources to treat allergic reactions of any degree of severity. In the clinical setting, biomarkers have been used and validated to enable an accurate diagnosis when combined with the clinical history, deferring the oral food challenge, whenever possible. In this review, we cover the tools available to support the diagnosis of food allergies and to predict food allergy resolution over time. We review the latest evidence on different testing modalities and how effective they are in guiding clinical decision-making in practice. We also evaluate predictive test cut-offs for the more common food allergens to try and provide guidance on when challenges might be most successful in determining oral tolerance in children.
\end{abstract}

\section{Hosted file}

Diagnostic paper - PAI 2020 Final saved.docx available at https://authorea.com/users/333787/ articles/459877-biomarkers-of-diagnosis-and-resolution-of-food-allergy 